\title{
OPTIMIZATION OF THE PRESERVED PROCESSING OF ALL PARTS OF THE GABIROBA FRUIT (CAMPOMANESIA ADAMANTIUM (CAMBESS.) O. BERG) USING RESPONSE SURFACE METHODOLOGY
}

\author{
Diana Lopes Silva ${ }^{1 凶}$, Gabriela Fonseca Leal ${ }^{2}$, Hermanny Matos Silva Sousa ${ }^{2}$, \\ Cecilia Marques Tenório Pereira ${ }^{3}$, Juliana Fonseca Moreira Silva ${ }^{4}$, \\ Joenes Mucci Peluzio², Glêndara Aparecida de Souza Martins² \\ ${ }^{1}$ Universidade Federal do Tocantins \\ Av. NS 15, 109 Norte, CEP 77010-090 Palmas, Tocantins, Brasil \\ ${ }^{2}$ Laboratório de Cinética e Modelagem de Processos, Engenharia de Alimentos, Universidade Federal do Tocantins \\ Av. NS 15, 109 Norte, CEP 77010-090 Palmas, Tocantins, Brasil \\ ${ }^{3}$ Laboratório de Microbiologia Aplicada, Universidade Federal do Tocantins \\ Av. NS 15, 109 Norte, CEP 77010-090 Palmas, Tocantins, Brasil \\ ${ }^{4}$ Laboratório de Microbiologia Geral e Aplicada, Universidade Federal do Tocantins \\ Av. NS 15, 109 Norte, CEP 77010-090 Palmas, Tocantins, Brasil
}

\begin{abstract}
Background. The Amazon region hosts several species of exotic fruits with great functional potential which are important sources of nutrients and biologically active compounds. These fruits are widely consumed by the local population, but unknown to the general population and the scientific community. In addition, the seasonality inherent in these fruits limits their consumption. In this context, the processing of food products is one of the main tools for improving sensory quality and increasing the useful life of fruits. This work aims to make use of all parts of the gabiroba fruit in the form of preserves with the replacement of commercial pectin by passion fruit albedo, as well as the characterization of the fruit and the processed product.

Methods. To optimize the preserves, a response surface methodology was used with a complete factorial design $2^{3}$ with 11 formulations and 3 independent variables, citric acid concentration, pulp/sugar ratio and albedo concentration, which were subjected to microbiological analysis, sensory analysis and physical-chemistry analysis. Results. The results showed that the gabiroba fruit has a low lipid content, $1.09 \%$, in addition to $51.20 \mathrm{~g} / 100 \mathrm{~g}$ vitamin C, $4.09 \%$ fiber, $79.83 \%$ moisture, $1.29 \%$ protein, $13.23 \%$ carbohydrates, $019 \%$ titratable acid and $0.47 \%$ ash. The result of the sensorial analysis indicated an optimal formulation with smaller amounts of citric acid and albedo and a lower pulp/sugar ratio.

Conclusion. The gabiroba fruit was suitable for its full use in preserve processing. Processed preserves showed desirable physical-chemical characteristics, with consumer preference for formulations with a lower pulp/sugar ratio indicating the impact of consumer unfamiliarity with the fruit on their decision to consume it.
\end{abstract}

Keywords: biodiversity, full use, optimization, new products

\section{INTRODUCTION}

Amazonian fruits are an important source of essential nutrients and biologically active compounds and their consumption can reduce the risk of acquiring chronic and degenerative diseases (Schulz and Chim, 2019).

『didilopess@uft.edu.br, phone +55 63984152542 
Native fruits are mostly sold in open markets with great popular acceptance. They have a distinctive flavor and can be consumed fresh or in the form of processed products. Processing of native fruits, in addition to adding value to the product, is a form of conservation, since many of these fruits are seasonal and highly perishable, which enables them to be used and consumed at other times of the year (Oliveira et al., 2020).

In this sense, gabiroba is an exotic fruit that is considered to be a functional food due to its nutritional properties, its content of vitamin C, carbohydrates, and water, and the presence of dietary fibers. Knowledge of the composition, physical-chemical and functional characteristics of native fruits is an essential tool in encouraging consumption, enabling the formulation of new products and making their use viable in the food industry (Goldoni et al., 2019).

Sweets can be classified according to consistency as creamy (or pasty) and cut sweet (preserves) (Aguiar et al., 2019). The preparation of preserves is understood as a method for preserving food, especially for fruit, which uses heat and sugar to increase the concentration of the mixture, reducing the water content and activity, thus creating unfavorable conditions for groups of microorganisms to develop (Dias et al., 2019). Preserves are products that have good chemical and microbiological stability (Tireki, 2017).

This study aimed to make full use of gabiroba fruits in the form of preserves with substitution of commercial pectin for passion fruit albedo, as well as the characterization of both the raw material and the processed product.

\section{MATERIALS AND METHODS}

\section{Fruit characterization}

The fruits were obtained from the Brazilian cerrado and selected according to their state of maturity and conservation. The fruits used were ripe and had average diameters of $18.3 \mathrm{~mm}$ and $17.2 \mathrm{~mm}$ longitudinal and transversal, respectively. For the characterization of the roughly 120 collected fruits, the following analsyes were performed: proximate composition, $\mathrm{pH}$, titratable total acidity and vitamin $\mathrm{C}$, all according to methodologies described by IAL - Instituto Adolfo Lutz (IAL, 2008).

\section{Preserve optimization}

For the processing of the preserves, eleven formulations were prepared according to the experimental design, Table 1. The formulation optimization used response surface methodology with a complete $2^{3}$ factorial design according to the methodology described by Box and Draper (1987), to evaluate the influence of citric acid concentration, pulp/sugar ratio and albedo concentration (independent variable) on the variables responses of aroma, flavor, texture, global impression, frequency of consumption and purchase intention. To validate the models, analysis of variance (ANOVA) was used to evaluate the significance (95\% confidence) of the regression using the $F$ test and the adjustment of the model to the experimental data using the coefficient of determination $\left(R^{2}\right)$.

The pulp used to process the preserves was from the whole fruit, therefore aiming at a higher yield and a sustainable process (full use of the fruit). The pulp was sent frozen to the Kinetic and Process Modeling Laboratory at the Federal University of Tocantins for storage until processing and characterization. Passion

Table 1. Experimental design $2^{3}$, for the preparation tests of gabiroba preserves

\begin{tabular}{ccccccc}
\hline & \multicolumn{3}{c}{ Coded variables } & \multicolumn{3}{c}{ Real variables } \\
\cline { 2 - 7 } Test & $X_{1}$ & $X_{2}$ & $X_{3}$ & $X_{1}, \%$ & $X_{2}, \mathrm{~g} / \mathrm{g}$ & $X_{3}, \%$ \\
\hline 1 & +1 & +1 & +1 & 1 & $60 / 40$ & 3 \\
2 & -1 & -1 & +1 & 0 & $40 / 60$ & 3 \\
3 & +1 & -1 & +1 & 1 & $40 / 60$ & 3 \\
4 & -1 & +1 & +1 & 0 & $60 / 40$ & 3 \\
5 & +1 & +1 & -1 & 1 & $60 / 40$ & 0 \\
6 & -1 & +1 & -1 & 0 & $60 / 40$ & 0 \\
7 & +1 & -1 & -1 & 1 & $40 / 60$ & 0 \\
8 & -1 & -1 & -1 & 0 & $40 / 60$ & 0 \\
9 & 0 & 0 & 0 & 0.5 & $50 / 50$ & 1.5 \\
10 & 0 & 0 & 0 & 0.5 & $50 / 50$ & 1.5 \\
11 & 0 & 0 & 0 & 0.5 & $50 / 50$ & 1.5 \\
\hline
\end{tabular}

$X_{1}$ - citric acid concentration, $\%, X_{2}-$ pulp/sugar ratio, $\mathrm{g} / \mathrm{g}, X_{3}-$ albedo concentration, $\%$. 
Silva, D. L., Fonseca Leal, G., Silva Sousa, H. M., Tenório Pereira, C. M., Moreira Silva, J. F., Peluzio, J. M., de Souza Martins, G. A. (2021). Optimization of the preserved processing of all parts of the gabiroba fruit (Campomanesia adamantium (Cambess.) O. Berg) using response surface methodology. Acta Sci. Pol. Technol. Aliment., 20(4), 399-416. http://dx.doi.org/10.17306/J.AFS.2021.0891

fruit albedo was used as a source of pectin, as described by Silva et al. (2012).

\section{Calculation of yields}

The yield of each formulation was calculated using equation 1.

$$
\% \text { preserves yield }=\frac{P_{C} \times 100}{P_{i}}
$$

where:

$P_{i}$ - weight of ingredients,

$P_{C}$ - weight of the preserves after processing.

\section{Determination of physicochemical characteristics of formulations}

The preserves produced were evaluated for their content of reducing and total sugars, yield, soluble solids, $\mathrm{pH}$, acidity, vitamin $\mathrm{C}$, ashes, and lipids according to methodologies described by IAL - Instituto AdoIfo Lutz (IAL, 2008). The color of the preserves was analyzed using the Konica Minolta colorimeter model CR400, D65 light source in color space $\left(L^{*}, a^{*}, b^{*}\right)$ following the coordinates of the CIELAB standards, in triplicate with three repetitions. The content of total soluble solids (TSS), ${ }^{\circ}$ Brix, was measured with the aid of a refractometer.

For texture profile analysis - TPA, the samples were placed in cylindrical jars of $50 \mathrm{~mm}$ in diameter and a Texturometer (TA.XT.Plus - Texture Analyzer) was used with P36R, pre-test, test and post-test speeds of $2 \mathrm{~mm} / \mathrm{s}$ and a distance of $26 \mathrm{~mm}$. The hardness and adhesion parameters were calculated and analyzed. For the quantification of lycopene, the methodology proposed by Nagata and Yamashita (1992) and Silva et al. (2013) was used. Analysis of variance (ANOVA) was used for the results of the physical-chemical analysis, which were evaluated by multiple comparisons of means using the Tukey test at 5\% $(P<0.05)$.

\section{Microbiological analysis}

The preserve formulations underwent microbiological analysis before being subjected to sensory analysis. The analyses were carried out to the standards required both by Resolução da Diretoria Colegiada - RDC $\mathrm{n}^{\mathrm{o}} 12$, which is currently in force (Brasil, 2001), and by $\mathrm{RDC} \mathrm{n}^{\circ} 331$, which will take effect in December 2020 (Brasil, 2019). To ensure the hygienic-sanitary quality of the products, an analysis of coagulase positive Staphylococci, Salmonella sp. and total coliforms was carried out in order to guarantee a food safe for human consumption. All microbiological analyses were performed according to the methodology described by Silva et al. (2017).

\section{Sensory analysis}

For the sensory analysis, the research project was submitted to analysis by the Ethics Committee of the Federal University of Tocantins, which was approved with an Ethical Appreciation Certificate number 93357718.3.0000.5519 and opinion number 3.096.189. For the formulation optimization stage, the samples were submitted to sensory acceptance tests by 36 untrained tasters for the attributes of aroma, flavor, texture, global impression, frequency of consumption and purchase intention, in addition to questions about their knowledge of the gabiroba fruit, using a hedonic scale of nine points. The sensory analysis procedures followed the methodology of Stone and Sidel (2004).

\section{RESULTS AND DISCUSSION}

\section{Fruit characterization}

The composition of the gabiroba fruit (peel and flesh) can be seen in Table 2. It can be classified as a perishable food due to its high moisture content, according to Vieira (2019), suggesting the need for processing to apply technologies that control this parameter.

The content of lipids and proteins found for gabiroba were low, however, in fruits and vegetables these constituents are available in reduced quantities (Goldoni et al., 2019; Negri et al., 2016). As for the fiber content, gabiroba is classified as a fruit with an average content of this constituent, according to Brazilian law (Brasil, 1998). The ash content observed is within the expected limits for this type of fruit (Paul and Duarte, 2012).

The carbohydrate content found in gabiroba makes it a high value fruit of this constituent in the parameters of RDC $\mathrm{n}^{\circ} 54$ (Brasil, 2012). In nutritional terms, the varied consumption of fruit represents an important source of carbohydrates.

The $\mathrm{pH}$ value of the fruit is an important factor in the processing of preserves and jellies as it is related to the formation of the gel, and should be between 3 and 3.5 
Silva, D. L., Fonseca Leal, G., Silva Sousa, H. M., Tenório Pereira, C. M., Moreira Silva, J. F., Peluzio, J. M., de Souza Martins, G. A. (2021). Optimization of the preserved processing of all parts of the gabiroba fruit (Campomanesia adamantium (Cambess.) O. Berg) using response surface methodology. Acta Sci. Pol. Technol. Aliment., 20(4), 399-416. http://dx.doi.org/10.17306/J.AFS.2021.0891

Table 2. Physico-chemical characteristics and centesimal composition of gabiroba pulp (C. adamantium), $\mathrm{g} \cdot 100 \mathrm{~g}^{-1}$

\begin{tabular}{lccc}
\hline Analyze $(n=3)$ & $\begin{array}{c}\text { Averages } \\
\pm \text { SD }\end{array}$ & $\begin{array}{c}\text { Analyze } \\
(n=3)\end{array}$ & $\begin{array}{c}\text { Averages } \\
\pm \text { SD }\end{array}$ \\
\hline Moisture, \% & $79.83 \pm 0.6$ & $\begin{array}{c}\text { vitamin C } \\
\text { mg of ascorbic } \\
\text { acid/100 g }\end{array}$ & $51.20 \pm 2.3$ \\
Lipids, \% & $1.09 \pm 0.23$ & pH & $4.2 \pm 0.45$ \\
Proteins, \% & $1.29 \pm 0.5$ & $\begin{array}{c}\text { total titratable } \\
\text { acidity, } \%\end{array}$ & $0.19 \pm 0.016$ \\
Crude fiber, \% & $4.09 \pm 0.5$ & & \\
Carbohydrates, \% & $13.23 \pm 0.25$ & & \\
Ashes, \% & $0.47 \pm 0.1$ & & \\
\hline
\end{tabular}

$n$ - number of replicates, $\mathrm{SD}-$ standard deviation, $\mathrm{pH}$ - hydrogenionic potential. for the production of this type of product (Bekele et al., 2020), so the value obtained indicates the need for adjustment.

The Ministry of Health recommends a daily intake of $60 \mathrm{mg}$ of vitamin $\mathrm{C}$ for adults (Brasil, 2003). In this context, a portion of approximately 120 grams of gabiroba exclusively meets the entire daily recommendation for intake of this nutrient.

\section{Preserve characterization}

Tables 3, 4 and 5 show the analysis of variance (ANOVA) for the physicochemical variables of the gabiroba preserve formulations.

As for coloring, there was a significant influence of the interactions between the sources of variation (Table 3 ) on the parameters $a^{*}, b^{*}$ and $L^{*}$ of the preserves. Although all formulations differ significantly from

Table 3. Analysis of variance results of color, yield, $\mathrm{pH}$ and total titratable acidity of gabiroba preserves

\begin{tabular}{|c|c|c|c|c|c|}
\hline Source & $\begin{array}{l}\text { Degrees of } \\
\text { freedom }\end{array}$ & Sum of squares & Mean square & $F$-value & $P$-value \\
\hline 1 & 2 & 3 & 4 & 5 & 6 \\
\hline \multicolumn{6}{|c|}{$a^{*}$} \\
\hline Acid & 2 & 1.07181 & 0.536 & 1.512 & 0.3982 \\
\hline Albedo & 2 & 0.08306 & 0.0415 & 0.117 & 0.8951 \\
\hline Pulp/sugar ratio & 2 & 0.495806 & 0.248 & 0.699 & 0.588 \\
\hline Acid* reason & 0 & 0.505794 & 0.536 & 1.512 & $0.000 *$ \\
\hline Acid* albedo & 0 & 2.966244 & 0.536 & 1.512 & $0.000^{*}$ \\
\hline Reason* albedo & 0 & 0.151044 & 0.248 & 0.699 & $0.000 *$ \\
\hline Acid* reason* albedo & 2 & 0.486256 & 0.243 & 0.686 & 0.5932 \\
\hline Error & 2 & 0.709067 & 0.354 & & \\
\hline Corrected total & 10 & 6.469073 & & & \\
\hline $\mathrm{CV} \%$ & 2 & 22.52 & & & \\
\hline Overall average & 2 & 2.6445 & & & \\
\hline \multicolumn{6}{|c|}{$b^{*}$} \\
\hline Acid & 2 & 6.600752 & 3.300 & 1388 & 0.4188 \\
\hline Albedo & 2 & 8.484002 & 4.242 & 1.784 & 0.3592 \\
\hline Pulp/sugar ratio & 2 & 15.01800 & 7.509 & 3.158 & 0.2405 \\
\hline
\end{tabular}


Silva, D. L., Fonseca Leal, G., Silva Sousa, H. M., Tenório Pereira, C. M., Moreira Silva, J. F., Peluzio, J. M., de Souza Martins, G. A. (2021). Optimization of the preserved processing of all parts of the gabiroba fruit (Campomanesia adamantium (Cambess.) O. Berg) using response surface methodology. Acta Sci. Pol. Technol. Aliment., 20(4), 399-416. http://dx.doi.org/10.17306/J.AFS.2021.0891

Table 3 - cont.

\begin{tabular}{|c|c|c|c|c|c|}
\hline 1 & 2 & 3 & 4 & 5 & 6 \\
\hline Acid* reason & 0 & 3.9763 & 3.300 & 1.388 & $0.000^{*}$ \\
\hline Acid* albedo & 0 & 8.65501 & 3.300 & 1.388 & $0.000 *$ \\
\hline Reason* albedo & 0 & 4.718351 & 7.509 & 3.158 & $0.000^{*}$ \\
\hline Acid* reason* albedo & 2 & 15.802552 & 7.901 & 3.322 & 0.2314 \\
\hline Error & 2 & 4.756267 & 2.378 & & \\
\hline Corrected total & 10 & 41.10142 & & & \\
\hline $\mathrm{CV} \%$ & 2 & 28.08 & & & \\
\hline Overall average & 2 & 5.4927 & & & \\
\hline \multicolumn{6}{|c|}{$L^{*}$} \\
\hline Acid & 2 & 127.5253 & 63.763 & 2923 & 0.2549 \\
\hline Albedo & 2 & 0.810758 & 0.405 & 0.019 & 0.9818 \\
\hline Pulp/sugar ratio & 2 & 0.255858 & 0.128 & 0.006 & 0.9942 \\
\hline Acid* reason & 0 & 0.156767 & 63.763 & 2.923 & $0.000^{*}$ \\
\hline Acid* albedo & 0 & 3.519867 & 63.763 & 2.923 & $0.000 *$ \\
\hline Reason* albedo & 0 & 70.541867 & 0.128 & 0.006 & $0.000^{*}$ \\
\hline Acid* reason* albedo & 2 & 96.203858 & 48.102 & 2.205 & 0.3120 \\
\hline Error & 2 & 43.630867 & 21.8154 & & \\
\hline Corrected total & 10 & 342.6452 & & & \\
\hline $\mathrm{CV} \%$ & 2 & 20.89 & & & \\
\hline Overall average & 2 & 22.36 & & & \\
\hline \multicolumn{6}{|c|}{ Yield } \\
\hline Acid & 2 & 236.9304 & 118.465 & 1692.360 & $0.0007 *$ \\
\hline Albedo & 2 & 249.8904 & 124.945 & 1784.932 & $0.0007 *$ \\
\hline Pulp/sugar ratio & 2 & 589.91545 & 294.957 & 4213.682 & $0.0003 *$ \\
\hline Acid* reason & 0 & 2.036104 & 118.465 & 1692.3603 & $0.0000^{*}$ \\
\hline Acid* albedo & 0 & 2.161054 & 118.465 & 1692.3603 & $0.0000^{*}$ \\
\hline Reason* albedo & 0 & 1.8203045 & 294.958 & 4213.682 & $0.0000^{*}$ \\
\hline Acid* reason* albedo & 2 & 232.315455 & 116.157 & 1659.936 & $0.0000^{*}$ \\
\hline Error & 2 & 0.140000 & 0.07000 & & \\
\hline Corrected total & 10 & 707.4454 & & & \\
\hline $\mathrm{CV} \%$ & 2 & 0.42 & & & \\
\hline Overall average & 2 & 62.6636 & & & \\
\hline
\end{tabular}


Silva, D. L., Fonseca Leal, G., Silva Sousa, H. M., Tenório Pereira, C. M., Moreira Silva, J. F., Peluzio, J. M., de Souza Martins, G. A. (2021). Optimization of the preserved processing of all parts of the gabiroba fruit (Campomanesia adamantium (Cambess.) O. Berg) using response surface methodology. Acta Sci. Pol. Technol. Aliment., 20(4), 399-416. http://dx.doi.org/10.17306/J.AFS.2021.0891

Table 3 - cont.

\begin{tabular}{|c|c|c|c|c|c|}
\hline 1 & 2 & 3 & 4 & 5 & 6 \\
\hline \multicolumn{6}{|c|}{$\mathrm{pH}$} \\
\hline Acid & 2 & 1.343788 & 0.682 & 201.568 & $0.0050^{*}$ \\
\hline Albedo & 2 & 0.263788 & 0.132 & 39.568 & $0.0247^{*}$ \\
\hline Pulp/sugar ratio & 2 & 0.298788 & 0.149 & 44.818 & $0.0218^{*}$ \\
\hline Acid* reason & 0 & 1.737878 & 0.682 & 201.568 & $0.0000^{*}$ \\
\hline Acid* albedo & 0 & 1.987877 & 0.682 & 201.568 & $0.0000^{*}$ \\
\hline Reason* albedo & 0 & 2.137878 & 0.149 & 44.818 & $0.0000 *$ \\
\hline Acid* reason* albedo & 2 & 0.238788 & 0.119 & 35.818 & $0.0272 *$ \\
\hline Error & 2 & 0.006667 & 0.003333 & & \\
\hline Corrected total & 10 & 1.565455 & & & \\
\hline $\mathrm{CV} \%$ & 2 & 1.21 & & & \\
\hline Overall average & 2 & 4.7636 & & & \\
\hline \multicolumn{6}{|c|}{ Titratable acidity } \\
\hline Acid & 2 & 210.6305 & 105.315 & 194.208 & $0.0052^{*}$ \\
\hline Albedo & 2 & 0.642717 & 0.321 & 0.593 & 0.6279 \\
\hline Pulp/sugar ratio & 2 & 2.472818 & 1.236 & 2.280 & 0.3049 \\
\hline Acid* reason & 0 & 5.84266 & 105.315 & 194.208 & $0.0000^{*}$ \\
\hline Acid* albedo & 0 & 4.07072 & 105.315 & 194.208 & $0.0000 *$ \\
\hline Reason* albedo & 0 & 8.0459 & 1.236 & 2.280 & $0.0000^{*}$ \\
\hline Acid* reason* albedo & 2 & 0.783645 & 0.392 & 0.723 & 0.5805 \\
\hline Error & 2 & 1.08 & 0.542282 & & \\
\hline Corrected total & 10 & 214.6149 & & & \\
\hline $\mathrm{CV} \%$ & 2 & 8.11 & & & \\
\hline Overall average & 2 & 9.081 & & & \\
\hline
\end{tabular}

* Significant at $P<0.05$.

each other $(P<0.05)$, in the Tukey test, formulations with higher amounts of citric acid showed a lighter color when compared to the others. However, formulations with higher concentrations of sugar (lower pulp/ sugar ratio) suffer more from the effects of reactions that occur during heating, such as the Maillard reaction (Curi et al., 2017). The gabiroba preserves have a greater tendency to be yellow in color, indicated by the values of $b^{*}$ (Table 7).
All sources of variation had a significant influence $(P<0.05)$ in relation to the $\mathrm{pH}$ of the preserves. The formulations with lower amounts of albedo and higher amounts of citric acid showed lower $\mathrm{pH}$ values. For total titratable acidity, only the variation of citric acid and the interactions between the sources of variation exerted a significant influence $(P<0.05)$ (Table 3$)$. In this context, the high values presented in some formulations can be justified, in addition to the addition 
Silva, D. L., Fonseca Leal, G., Silva Sousa, H. M., Tenório Pereira, C. M., Moreira Silva, J. F., Peluzio, J. M., de Souza Martins, G. A. (2021). Optimization of the preserved processing of all parts of the gabiroba fruit (Campomanesia adamantium (Cambess.) O. Berg) using response surface methodology. Acta Sci. Pol. Technol. Aliment., 20(4), 399-416. http://dx.doi.org/10.17306/J.AFS.2021.0891

Table 4. Analysis of variance results of the physicochemical variables (vitamin C, lipids, total soluble solids, lycopene, ashes) of gabiroba preserves

\begin{tabular}{|c|c|c|c|c|c|}
\hline Source & $\begin{array}{l}\text { Degrees of } \\
\text { freedom }\end{array}$ & Sum of squares & Mean square & $F$-value & $P$-value \\
\hline 1 & 2 & 3 & 4 & 5 & 6 \\
\hline \multicolumn{6}{|c|}{ Vitamin C } \\
\hline Acid & 2 & 165.605152 & 82.802 & 0.988 & 0.5030 \\
\hline Albedo & 2 & 162.005152 & 81.002 & 0.967 & 0.5085 \\
\hline Pulp/sugar ratio & 2 & 1.880152 & 0.94 & 0.011 & 0.9889 \\
\hline Acid* reason & 0 & 1.620151 & $82.802 *$ & 0.988 & $0.0000^{*}$ \\
\hline Acid* albedo & 0 & 131.044848 & $82.802 *$ & 0.988 & $0.0000^{*}$ \\
\hline Reason* albedo & 0 & 1.4801515 & $0.94 *$ & 0.011 & $0.0000^{*}$ \\
\hline Acid* reason* albedo & 2 & 1.820152 & 0.91 & 0.0 & 0.9893 \\
\hline Error & 2 & 167.606667 & 83.803 & & \\
\hline Corrected total & 10 & 626.861818 & & & \\
\hline $\mathrm{CV} \%$ & 2 & 23.61 & & & \\
\hline Overall average & 2 & 38.7727 & & & \\
\hline \multicolumn{6}{|c|}{ Lipids } \\
\hline Acid & 2 & 0.001722 & 0.000861 & 0.086 & 0.9210 \\
\hline Albedo & 2 & 0.005922 & 0.002961 & 0.295 & 0.7721 \\
\hline Pulp/sugar ratio & 2 & 0.015022 & 0.007511 & 0.749 & 0.5719 \\
\hline Acid* reason & 0 & 1.3969 & 0.000861 & 0.086 & $0.0000^{*}$ \\
\hline Acid* albedo & 0 & 0.017603 & 0.000861 & 0.086 & $0.0000^{*}$ \\
\hline Reason* albedo & 0 & 0.015703 & 0.007511 & 0.749 & $0.0000^{*}$ \\
\hline Acid* reason* albedo & 2 & 0.057522 & 0.028761 & 2.867 & 0.2586 \\
\hline Error & 2 & 0.020067 & 0.010033 & & \\
\hline Corrected total & 10 & & & & \\
\hline $\mathrm{CV} \%$ & 2 & 26.94 & & & \\
\hline Overall average & 2 & 0.3718 & & & \\
\hline \multicolumn{6}{|c|}{ Total soluble solids } \\
\hline Acid & 2 & 37.775152 & 18.887 & 354.142 & $0.0030^{*}$ \\
\hline Albedo & 2 & 37.775152 & 18.887 & 354.1 & $0.0030^{*}$ \\
\hline Pulp/sugar ratio & 2 & 77.775152 & 38.887 & 729.142 & $0.0015^{*}$ \\
\hline Acid* reason & 0 & 2.927515 & 18.887 & 354.1 & $0.0000^{*}$ \\
\hline Acid* albedo & 0 & 5.275151 & 18.887 & 354.1 & $0.0000^{*}$ \\
\hline
\end{tabular}


Silva, D. L., Fonseca Leal, G., Silva Sousa, H. M., Tenório Pereira, C. M., Moreira Silva, J. F., Peluzio, J. M., de Souza Martins, G. A. (2021). Optimization of the preserved processing of all parts of the gabiroba fruit (Campomanesia adamantium (Cambess.) O. Berg) using response surface methodology. Acta Sci. Pol. Technol. Aliment., 20(4), 399-416. http://dx.doi.org/10.17306/J.AFS.2021.0891

Table 4 - cont.

\begin{tabular}{|c|c|c|c|c|c|}
\hline 1 & 2 & 3 & 4 & 5 & 6 \\
\hline Reason* albedo & 0 & 2.927551 & 38.887 & 729.142 & $0.0000^{*}$ \\
\hline Acid* reason* albedo & 2 & 61.775152 & 30.887 & 579.142 & $0.0019^{*}$ \\
\hline Error & 2 & 0.106667 & 0.053333 & & \\
\hline Corrected total & 10 & 151.381818 & & & \\
\hline CV\% & 2 & 0.32 & & & \\
\hline Overall average & 2 & 72.87 & & & \\
\hline \multicolumn{6}{|c|}{ Lycopene } \\
\hline Acid & 2 & 14.933439 & 7.467 & 0.675 & 0.5972 \\
\hline Albedo & 2 & 21.523091 & 10.626 & 0.106 & 0.9041 \\
\hline Pulp/sugar ratio & 2 & 3.341975 & 1.671 & 0.474 & $0.0000 *$ \\
\hline Acid* reason & 0 & 5.148814 & $7.467 *$ & 0.474 & $0.0000 *$ \\
\hline Acid* albedo & 0 & 1.755274 & $7.467 *$ & 0.106 & $0.0000 *$ \\
\hline Reason* albedo & 0 & 2.180177 & $1.671^{*}$ & 0.078 & 0.9274 \\
\hline Acid* reason* albedo & 2 & 2.465943 & 1.233 & & \\
\hline Error & 2 & 31.508952 & 15.754476 & & \\
\hline Corrected total & 10 & 82.587665 & & & \\
\hline $\mathrm{CV} \%$ & 2 & 71.53 & & & \\
\hline Overall average & 2 & 5.5494 & & & \\
\hline \multicolumn{6}{|c|}{ Ashes } \\
\hline Acid & 2 & 0.086983 & 0.043492 & 186.393 & $0.0054 *$ \\
\hline Albedo & 2 & 0.060733 & 0.030367 & 130.143 & $0.0075^{*}$ \\
\hline Pulp/sugar ratio & 2 & 0.047933 & 0.023967 & 102.714 & $0.0094 *$ \\
\hline Acid* reason & 0 & 0.073117 & 0.043492 & 186.393 & $0.0000 *$ \\
\hline Acid* albedo & 0 & 0.073117 & 0.043492 & 186.393 & $0.0000^{*}$ \\
\hline Reason* albedo & 0 & 0.102867 & 0.023967 & 102.714 & $0.0000 *$ \\
\hline Acid* reason* albedo & 2 & 0.132983 & 0.066492 & 284.964 & $0.0036^{*}$ \\
\hline Error & 2 & 0.000467 & 0.000233 & & \\
\hline Corrected total & 10 & 0.578200 & & & \\
\hline $\mathrm{CV} \%$ & 2 & 8.04 & & & \\
\hline Overall average & 2 & 0.19 & & & \\
\hline
\end{tabular}

*Significant at $P<0.05$. 
Silva, D. L., Fonseca Leal, G., Silva Sousa, H. M., Tenório Pereira, C. M., Moreira Silva, J. F., Peluzio, J. M., de Souza Martins, G. A. (2021). Optimization of the preserved processing of all parts of the gabiroba fruit (Campomanesia adamantium (Cambess.) O. Berg) using response surface methodology. Acta Sci. Pol. Technol. Aliment., 20(4), 399-416. http://dx.doi.org/10.17306/J.AFS.2021.0891

Table 5. Analysis of variance of physicochemical variables (total sugar, reducing sugar, moisture, hardness, adhesiveness) of gabiroba preserves

\begin{tabular}{|c|c|c|c|c|c|}
\hline Source & $\begin{array}{l}\text { Degrees of } \\
\text { freedom }\end{array}$ & Sum of squares & Mean square & $F$-value & $P$-value \\
\hline 1 & 2 & 3 & 4 & 5 & 6 \\
\hline \multicolumn{6}{|c|}{ Total sugars } \\
\hline Acid & 2 & 109.638333 & 54.819 & 6.454 & 0.1342 \\
\hline Albedo & 2 & 121.51833 & 60.759 & 7.154 & 0.1226 \\
\hline Pulp/sugar ratio & 2 & 159.79833 & 79.899 & 9.407 & 0.0961 \\
\hline Acid* reason & 0 & 9.46133 & 54.819 & 6.454 & $0.0000^{*}$ \\
\hline Acid* albedo & 0 & 1.003733 & 54.819 & 6.454 & $0.0000^{*}$ \\
\hline Reason* albedo & 0 & 6.67733 & 79.899 & 9.407 & $0.0000^{*}$ \\
\hline Acid* reason* albedo & 2 & 130.0383 & 65.019 & 7.655 & 0.1155 \\
\hline Error & 2 & 16.98667 & 8.49333 & & \\
\hline Corrected total & 10 & 276.220000 & & & \\
\hline $\mathrm{CV} \%$ & 2 & 4.58 & & & \\
\hline Overall average & 2 & 63.7000 & & & \\
\hline \multicolumn{6}{|c|}{ Reducing sugars } \\
\hline Acid & 2 & 264.342718 & 132.171 & 51.9799 & $0.0189 *$ \\
\hline Albedo & 2 & 35.532718 & 17.766 & 6.987 & 0.1252 \\
\hline Pulp/sugar ratio & 2 & 37.392718 & 18.696 & 7.353 & 0.1197 \\
\hline Acid* reason & 0 & 9.229782 & 132.171 & 51.9779 & $0.0000^{*}$ \\
\hline Acid* albedo & 0 & 1.132021 & 132.171 & 51.979 & $0.0000^{*}$ \\
\hline Reason* albedo & 0 & 7.579782 & 18.696 & 7.353 & $0.0000^{*}$ \\
\hline Acid* reason* albedo & 2 & 38.382718 & 19.191 & 7.547 & 0.1170 \\
\hline Error & 2 & 5.085600 & 2.542800 & & \\
\hline Corrected total & 10 & 386.225818 & & & \\
\hline $\mathrm{CV} \%$ & 2 & 14.69 & & & \\
\hline Overall average & 2 & 10.8527 & & & \\
\hline \multicolumn{6}{|c|}{ Moisture } \\
\hline Acid & 2 & 25.4588 & 12.729 & 1.436 & 0.4105 \\
\hline Albedo & 2 & 1.968888 & 0.984 & 0.1111 & 0.900 \\
\hline Pulp/sugar ratio & 2 & 81.445438 & 40.722 & 4.595 & 0.1787 \\
\hline Acid* reason & 0 & 0.574162 & 12.729 & 1.436 & $0.0000^{*}$ \\
\hline Acid* albedo & 0 & 1.360412 & 12.729 & 1.436 & $0.0000^{*}$ \\
\hline
\end{tabular}


Silva, D. L., Fonseca Leal, G., Silva Sousa, H. M., Tenório Pereira, C. M., Moreira Silva, J. F., Peluzio, J. M., de Souza Martins, G. A. (2021). Optimization of the preserved processing of all parts of the gabiroba fruit (Campomanesia adamantium (Cambess.) O. Berg) using response surface methodology. Acta Sci. Pol. Technol. Aliment., 20(4), 399-416. http://dx.doi.org/10.17306/J.AFS.2021.0891

Table 5 - cont.

\begin{tabular}{|c|c|c|c|c|c|}
\hline 1 & 2 & 3 & 4 & 5 & 6 \\
\hline Reason* albedo & 0 & 3.876562 & 40.722 & 4.595 & $0.0000^{*}$ \\
\hline Acid* reason* albedo & 2 & 1.9112638 & 0.956319 & 0.108 & 0.9026 \\
\hline Error & 2 & 17.725067 & 8.86253 & & \\
\hline Corrected total & 10 & 134.322055 & & & \\
\hline CV\% & 2 & 20.33 & & & \\
\hline Overall average & 2 & 14.6463 & & & \\
\hline \multicolumn{6}{|c|}{ Hardness } \\
\hline Acid & 2 & 179.166506 & 89.583 & 1325.650 & $0.0009^{*}$ \\
\hline Albedo & 2 & 173.187078 & 86.593 & 1281.409 & $0.0009 *$ \\
\hline Pulp/sugar ratio & 2 & 182.710086 & 91.355 & 1351.869 & $0.0009 *$ \\
\hline Acid* reason & 0 & 118.699547 & 89.583 & 1325.650 & $0.0000^{*}$ \\
\hline Acid* albedo & 0 & 84.041555 & 89.583 & 1325.650 & $0.0000^{*}$ \\
\hline Reason* albedo & 0 & 82.337855 & 91.355 & 1351.869 & $0.0000^{*}$ \\
\hline Acid* reason* albedo & 2 & 180.76557 & 90.383 & 1337.482 & $0.0009 *$ \\
\hline Error & 2 & 0.135154 & 0.06757 & & \\
\hline Corrected total & 10 & 1001.043358 & & & \\
\hline $\mathrm{CV} \%$ & 2 & 7.38 & & & \\
\hline Overall average & 2 & 3.52 & & & \\
\hline \multicolumn{6}{|c|}{ Adhesiveness } \\
\hline Acid & 2 & 0.396670 & 0.198335 & 200.665 & $0.0050^{*}$ \\
\hline Albedo & 2 & 0.385790 & 0.192895 & 195.161 & $0.0052 *$ \\
\hline Pulp/sugar ratio & 2 & 0.432320 & 0.216160 & 218.699 & $0.0046^{*}$ \\
\hline Acid* reason & 0 & 0.134914 & 0.198335 & 200.665 & $0.0000^{*}$ \\
\hline Acid* albedo & 0 & 0.086404 & 0.198335 & 200.665 & $0.0000^{*}$ \\
\hline Reason* albedo & 0 & 0.079434 & 0.216160 & 218.699 & $0.0000^{*}$ \\
\hline Acid* reason* albedo & 2 & 0.481600 & 0.240800 & 243.629 & $0.0042 *$ \\
\hline Error & 2 & 0.001977 & 0.000988 & & \\
\hline Corrected total & 10 & 1.999109 & & & \\
\hline $\mathrm{CV} \%$ & 2 & 13.63 & & & \\
\hline Overall average & 2 & 0.2306 & & & \\
\hline
\end{tabular}

*Significant at $P<0.05$. 
Silva, D. L., Fonseca Leal, G., Silva Sousa, H. M., Tenório Pereira, C. M., Moreira Silva, J. F., Peluzio, J. M., de Souza Martins, G. A. (2021). Optimization of the preserved processing of all parts of the gabiroba fruit (Campomanesia adamantium (Cambess.) O. Berg) using response surface methodology. Acta Sci. Pol. Technol. Aliment., 20(4), 399-416. http://dx.doi.org/10.17306/J.AFS.2021.0891

Table 6. Analysis of variance results of sensory analysis for fitted models

\begin{tabular}{|c|c|c|c|c|c|}
\hline Source & Degrees of freedom & Sum of squares & Mean square & $F$-value & $P$-value \\
\hline \multicolumn{6}{|c|}{ Aroma } \\
\hline Regression & 7 & 2.79901 & 0.399 & 0.80 & 0.64 \\
\hline Lack-of-fit & 1 & 0.00781 & 0.00781 & 0.01 & 0.928 \\
\hline Pure error & 2 & 1.5000 & 0.7500 & & \\
\hline Total & 10 & 4.30682 & & & \\
\hline \multicolumn{6}{|c|}{ Flavor } \\
\hline Regression & 7 & 14.6884 & 2.09835 & 14.05 & $0.026^{*}$ \\
\hline Lack-of-fit & 1 & 0.2812 & 0.28125 & 3.37 & 0.208 \\
\hline Pure error & 2 & 0.1667 & 0.08333 & & \\
\hline Total & 10 & 15.1364 & & & \\
\hline \multicolumn{6}{|c|}{ Texture } \\
\hline Regression & 7 & 22.4697 & 3.2100 & 0.70 & 0.685 \\
\hline Lack-of-fit & 1 & 12.5000 & 12.5000 & 21.43 & $0.044^{*}$ \\
\hline Pure error & 2 & 1.1667 & 0.5833 & & \\
\hline Total & 10 & 36.1364 & & & \\
\hline \multicolumn{6}{|c|}{ Global impression } \\
\hline Regression & 7 & 9.4091 & 1.34416 & 4.03 & 0.140 \\
\hline Lack-of-fit & 1 & 0.5000 & 0.5000 & 2.00 & 0.293 \\
\hline Pure error & 2 & 0.5000 & 0.2500 & & \\
\hline Total & 10 & 10.4091 & & & \\
\hline \multicolumn{6}{|c|}{ Frequency of consumption } \\
\hline Regression & 7 & 3.21117 & 0.45874 & 0.63 & 0.726 \\
\hline Lack-of-fit & 1 & 0.03125 & 0.03125 & 0.03 & 0.881 \\
\hline Pure error & 2 & 2.16667 & 1.08333 & & \\
\hline Total & 10 & 5.40909 & & & \\
\hline \multicolumn{6}{|c|}{ Purchase intention } \\
\hline Regression & 7 & 4.43561 & 0.63366 & 1.47 & 0.408 \\
\hline Lack-of-fit & 1 & 0.12500 & 0.12500 & 0.21 & 0.689 \\
\hline Pure error & 2 & 1.16667 & 0.58333 & & \\
\hline Total & 10 & 5.72727 & & & \\
\hline
\end{tabular}

*Significant at $P<0.05$. 
of citric acid, due to the acidity of the gabiroba pulp itself.

In relation to the total soluble solids of the preserves (Table 4), all sources of variation had a significant influence $(P<0.05)$. Gabiroba preserves with a lower pulp/sugar ratio and higher amounts of albedo and acid had a higher content of total soluble solids (Table 7). Regarding total sugars, the more acidic formulations showed a higher concentration (Table 8) and, in this process, tended to reduce the gelation time and the time to reach the desired concentration, minimizing hydrolytic reactions. During cooking, sucrose in an acid medium undergoes hydrolysis and is partially broken down into reducing sugars.

The variation of citric acid and the interactions between the sources of variation exerted a significant influence $(P<0.05)$ on reducing sugars. Preserves with higher amounts of citric acid had a higher content of reducing sugars (Table 8). During cooking, sucrose in an acid medium undergoes hydrolysis, being partially broken down into reducing sugars such as glucose and fructose. In addition, reducing sugars are of great importance in the manufacture of jellies as they add shine to the product and can reduce and/or delay the crystallization of sucrose, which is reflected in the reduction of their sweetness level (Tireki, 2017).
The vitamin $\mathrm{C}$ content in gabiroba preserves was significantly influenced only by the interactions between the sources of variation $(P<0.05)$ (Table 4$)$, indicating that formulations with higher amounts of citric acid, less albedo and less pulp/sugar ratio have higher levels of vitamin C (Table 8). Formulations with a lower pulp/sugar ratio need less cooking time to achieve the required soluble solids content, which can then justify the higher vitamin $\mathrm{C}$ content given its sensitivity to degradation during heating.

For the lycopene values found in the formulations it was observed that preserves that had a lower pulp/ sugar ratio and a higher amount of albedo had higher concentrations of lycopene (Table 8). Fruit-derived products contain a lycopene content higher than fresh fruit due to the concentration stage during its processing (Lemos et al., 2011). The increase in the content of lycopene in these products occurs due to the action of temperature, which causes the rupture of cell walls, releasing it from the chromoplasts, making free lycopene available (Correia et al., 2008).

The formulations that had the lowest pulp/sugar ratio (highest amount of sugar) were the ones that had the better yield (Table 8). In this context, Silva et al. (2012) stated that the greater the amount of sugar present in the preserves, the greater the yield, since

Table 7. Color, $\mathrm{pH}$, total soluble solids and total titratable acidity of gabiroba preserves

\begin{tabular}{ccccccc}
\hline Formulation & $a^{*}$ & $b^{*}$ & $L^{*}$ & $\mathrm{pH}$ & Total soluble solids, ${ }^{\mathrm{o}}$ Brix & Titratable acidity, \% \\
\hline 1 & $3.44^{\mathrm{de}}$ & $3.66^{\mathrm{f}}$ & $26.6^{\mathrm{gh}}$ & $4.6^{\mathrm{d}}$ & $72^{\mathrm{bc}}$ & $14.5^{\mathrm{ef}}$ \\
2 & $1.67^{\mathrm{ab}}$ & $3.4^{\mathrm{c}}$ & $11.04^{\mathrm{a}}$ & $5.3^{\mathrm{g}}$ & $70^{\mathrm{ab}}$ & $4.14^{\mathrm{ab}}$ \\
3 & $3.57^{\mathrm{de}}$ & $7.5^{\mathrm{i}}$ & $26.95^{\mathrm{h}}$ & $4.4^{\mathrm{b}}$ & $80^{\mathrm{e}}$ & $13.7^{\mathrm{e}}$ \\
4 & $1.45^{\mathrm{a}}$ & $3.03^{\mathrm{a}}$ & $23.86^{\mathrm{e}}$ & $5.4^{\mathrm{h}}$ & $73^{\mathrm{cd}}$ & $4.2^{\mathrm{ab}}$ \\
5 & $2.54^{\mathrm{c}}$ & $5.84^{\mathrm{g}}$ & $26.85^{\mathrm{h}}$ & $4.7^{\mathrm{e}}$ & $70^{\mathrm{ab}}$ & $7.1^{\mathrm{f}}$ \\
6 & $2.05^{\mathrm{bc}}$ & $3.30^{\mathrm{b}}$ & $12.93^{\mathrm{b}}$ & $5.1^{\mathrm{f}}$ & $72^{\mathrm{bc}}$ & $13.9^{\mathrm{e}}$ \\
7 & $2.33^{\mathrm{c}}$ & $5.27^{\mathrm{ev}}$ & $25.22^{\mathrm{f}}$ & $4.2^{\mathrm{a}}$ & $75^{\mathrm{d}}$ & $3.15^{\mathrm{a}}$ \\
8 & $3.83^{\mathrm{e}}$ & $8.68^{\mathrm{kv}}$ & $25.86^{\mathrm{fg}}$ & $5.1^{\mathrm{f}}$ & $80^{\mathrm{e}}$ & $7.8^{\mathrm{c}}$ \\
10 & $2.05^{\mathrm{c}}$ & $4.82^{\mathrm{d}}$ & $17.5^{\mathrm{c}}$ & $4.6^{\mathrm{d}}$ & $69.6^{\mathrm{a}}$ & $8.9^{\mathrm{cd}}$ \\
11 & $3.11^{\mathrm{d}}$ & $7.74^{\mathrm{j}}$ & $22.31^{\mathrm{d}}$ & $4.5^{\mathrm{c}}$ & $70^{\mathrm{ab}}$ & $70^{\mathrm{ab}}$ \\
\hline
\end{tabular}

Means followed by equal letters do not differ, by the Tukey's test, at 5\% probability. 
Silva, D. L., Fonseca Leal, G., Silva Sousa, H. M., Tenório Pereira, C. M., Moreira Silva, J. F., Peluzio, J. M., de Souza Martins, G. A. (2021). Optimization of the preserved processing of all parts of the gabiroba fruit (Campomanesia adamantium (Cambess.) O. Berg) using response surface methodology. Acta Sci. Pol. Technol. Aliment., 20(4), 399-416. http://dx.doi.org/10.17306/J.AFS.2021.0891

Table 8. Total sugar, reducing sugar, vitamin C, lycopene, yield and ashes of gabiroba preserves

\begin{tabular}{|c|c|c|c|c|c|c|}
\hline Formulation & $\begin{array}{c}\text { Total sugars } \\
\%\end{array}$ & $\begin{array}{c}\text { Reducing sugars } \\
\%\end{array}$ & $\begin{array}{l}\text { Vitamin C } \\
\mathrm{mg} / 100 \mathrm{~g}\end{array}$ & $\begin{array}{l}\text { Lycopene } \\
\mu \mathrm{g} / 100 \mathrm{~mL}\end{array}$ & $\begin{array}{l}\text { Yield } \\
\%\end{array}$ & $\begin{array}{c}\text { Ashes } \\
\%\end{array}$ \\
\hline 1 & $70.3^{c}$ & $13.1^{\mathrm{e}}$ & $34.6^{\mathrm{e}}$ & $5.07^{\mathrm{e}}$ & $53.7^{\mathrm{b}}$ & $0.10^{\mathrm{c}}$ \\
\hline 2 & $69.6^{\mathrm{bc}}$ & $6.0^{\mathrm{ab}}$ & $33.1^{\mathrm{a}}$ & $11.23^{\mathrm{k}}$ & $57.8^{c}$ & $0.19^{i}$ \\
\hline 3 & $66.1^{\mathrm{bc}}$ & $26.3^{\mathrm{f}}$ & $34.4^{\mathrm{d}}$ & $5.74^{\mathrm{h}}$ & $67.3^{\mathrm{d}}$ & $0.12^{\mathrm{d}}$ \\
\hline 4 & $62.7^{\mathrm{abc}}$ & $6.2^{\mathrm{ab}}$ & $34.1^{\mathrm{c}}$ & $7.43^{\mathrm{i}}$ & $54.1^{\mathrm{b}}$ & $0.05^{\mathrm{a}}$ \\
\hline 5 & $59.2^{\mathrm{ab}}$ & $14.8^{\mathrm{e}}$ & $51.4^{\mathrm{g}}$ & $5.23^{\mathrm{f}}$ & $51.2^{\mathrm{a}}$ & $0.05^{\mathrm{a}}$ \\
\hline 6 & $59.1^{\mathrm{ab}}$ & $8.2^{\mathrm{c}}$ & $34.4^{\mathrm{d}}$ & $4.07^{\mathrm{d}}$ & $53.6^{\mathrm{b}}$ & $0.90^{\mathrm{j}}$ \\
\hline 7 & $70.9^{c}$ & $14.8^{\mathrm{e}}$ & $51.8^{\mathrm{h}}$ & $2.22^{\mathrm{b}}$ & $71.5^{\mathrm{f}}$ & $0.12^{\mathrm{e}}$ \\
\hline 8 & $66.5^{\mathrm{abc}}$ & $4.9^{\mathrm{a}}$ & $34.4^{\mathrm{d}}$ & $5.61^{\mathrm{g}}$ & $69.8^{\mathrm{e}}$ & $0.07^{\mathrm{b}}$ \\
\hline 9 & $56.7^{\mathrm{a}}$ & $7.50^{\mathrm{bc}}$ & $50.0^{\mathrm{f}}$ & $2.97^{\mathrm{c}}$ & $70.0^{\mathrm{e}}$ & $0.16^{\mathrm{g}}$ \\
\hline 10 & $62.1^{\mathrm{abc}}$ & $7.38^{\mathrm{bc}}$ & $34.4^{\mathrm{d}}$ & $2.11^{\mathrm{a}}$ & $70.4^{\mathrm{e}}$ & $0.18^{\mathrm{h}}$ \\
\hline 11 & $57.5^{\mathrm{a}}$ & $10.2^{\mathrm{d}}$ & $33.9^{\mathrm{b}}$ & $9.37^{\mathrm{j}}$ & $69.9^{\mathrm{e}}$ & $0.15^{\mathrm{f}}$ \\
\hline
\end{tabular}

Means followed by equal letters do not differ, by the Tukey's test, at 5\% probability.

Table 9. Lipids, moisture, hardness and adhesiveness of gabiroba preserves

\begin{tabular}{|c|c|c|c|c|}
\hline Formulation & Lipids, $\%$ & Moisture, \% & Hardness, $\mathrm{N}$ & Adhesiveness, $\mathrm{N}$ \\
\hline 1 & $0.53^{\mathrm{j}}$ & $15.6^{\mathrm{g}}$ & $1.17^{\mathrm{i}}$ & $-0.16^{\mathrm{b}}$ \\
\hline 2 & $0.44^{\mathrm{g}}$ & $13.2^{\mathrm{f}}$ & $33.66^{\mathrm{k}}$ & $-1.57^{\mathrm{a}}$ \\
\hline 3 & $0.19^{\mathrm{a}}$ & $11.3^{\mathrm{b}}$ & $0.146^{\mathrm{c}}$ & $-0.09^{\mathrm{h}}$ \\
\hline 4 & $0.45^{\mathrm{h}}$ & $18.3^{\mathrm{j}}$ & $0.094^{\mathrm{a}}$ & $-0.07^{\mathrm{j}}$ \\
\hline 5 & $0.32^{\mathrm{d}}$ & $16.0^{\mathrm{h}}$ & $0.47^{\mathrm{g}}$ & $-0.12^{\mathrm{h}}$ \\
\hline 6 & $0.38^{\mathrm{f}}$ & $22.2^{\mathrm{k}}$ & $0.27^{\mathrm{d}}$ & $-0.13^{\mathrm{g}}$ \\
\hline 7 & $0.5^{\mathrm{i}}$ & $9.6^{\mathrm{a}}$ & $0.43^{\mathrm{f}}$ & $-0.16^{\mathrm{c}}$ \\
\hline 8 & $0.22^{\mathrm{b}}$ & $12.7^{\mathrm{d}}$ & $1.41^{\mathrm{j}}$ & $-0.136^{\mathrm{f}}$ \\
\hline 9 & $0.25^{\mathrm{c}}$ & $17.4^{\mathrm{i}}$ & $0.102^{\mathrm{b}}$ & $-0.07^{\mathrm{j}}$ \\
\hline 10 & $0.45^{\mathrm{h}}$ & $13.1^{\mathrm{e}}$ & $0.3526^{\mathrm{d}}$ & $-0.152^{\mathrm{e}}$ \\
\hline 11 & $0.36^{\mathrm{e}}$ & $11.73^{\mathrm{c}}$ & $0.6218^{\mathrm{h}}$ & $-0.159^{d}$ \\
\hline
\end{tabular}

Means followed by equal letters do not differ, by the Tukey's test, at $5 \%$ probability.

the gelation time is inversely proportional to the sugar concentration.

Regarding the ash content, formulations with a higher pulp/sugar ratio and lower amounts of albedo and acid obtained the highest ash contents (Table 8). The interactions between the sources of variation exerted a significant influence $(P<0.05)$ on the lipid content, indicating that the preserves with the highest 
pulp/sugar ratio showed significant amounts of these constituents (Table 9). Regarding these constituents, the observed response can be justified by the higher pulp/sugar ratio in the formulation as ash and lipids migrated from the pulp to the formulation of the jam, with no degradation of this content during processing.

In the results of the texture analysis, the causes of variation had a significant influence $(P<0.05)$ in relation to the hardness of the preserves (Table 5). Higher levels of albedo resulted in harder formulations. The formulations with higher pulp/sugar ratios and higher amounts of citric acid obtained the highest values for adhesiveness (Table 9). Formulations with a higher pulp/sugar ratio have higher amounts of organic acids from fruits, which directly influences the content of soluble solids in the product and, consequently, increases the adhesiveness (Dias et al., 2019).

\section{Microbiological analysis}

For the counting of molds and yeasts, all formulations analyzed showed values below those recommended by RDC $n^{\circ}$ 12/2001 and RDC $n^{\circ} 331 / 2019$ $\left(<10^{4} \mathrm{UFC} \cdot \mathrm{g}^{-1}\right)$. Salmonella sp. was not detected in any of the analyzed formulations, indicating that the results are in accordance with the CNNPA $n^{\circ} 12$, 1978, which establishes the absence of the microorganism in $25 \mathrm{~g}$ of sample. In the analysis of coagulase positive Staphylococci, all formulations were successful $<10 \mathrm{UFC} \cdot \mathrm{g}^{-1}$; and for total coliforms, values $<3$ NMP $\cdot \mathrm{g}^{-1}$ were obtained, indicating that the microbiological analyses met the limits established by the legislation and, therefore, the product was microbiologically fit for consumption.

\section{Sensory analysis}

The tasters were asked about their knowledge of the gabiroba fruit and $25 \%$ reported knowing the fruit, but had never consumed it, $30.55 \%$ reported not knowing the fruit, and $22.22 \%$ had already consumed it.

The regression equation for the aroma of preserves is:

$$
\begin{gathered}
\text { Aroma }=16.98-21.0 X_{1}-2.12 X_{2}-0.71 X_{3}+ \\
+11.22 X_{1} X_{1}+0.73 X_{1} X_{2}+0.722 X_{1} X_{3}+0.081 X_{2} X_{3} \\
\left(R^{2}=0.6499\right)
\end{gathered}
$$

It was observed that the concentration of citric acid $\left(X_{1}\right)$, pulp/sugar ratio $\left(X_{2}\right)$ and the albedo concentration
$\left(X_{3}\right)$ had a negative linear effect in relation to the aroma. The interactions between the factors had a positive linear effect. ANOVA for the model is provided in Table 6. The regression model adjusted to the experimental results of the aroma shows insignificant values for the sources. Figure 1 shows the behavior of the aroma attributes of the formulations, in which the highest scores were for the lowest concentrations of acid, albedo and pulp/sugar ratio.

For flavor (Fig. 2) it was observed that the highest scores were attributed to formulations with lower concentrations of albedo, citric acid and pulp/sugar ratio. The regression equation for the flavor of preserves is:

$$
\begin{gathered}
\text { Flavor }=5.68+16.64 X_{1}-2.30 X_{2}-2.294 X_{3}- \\
-15.36 X_{1} X_{1}+1.45 X_{1} X_{2}+0.778 X_{1} X_{3}+0.806 X_{2} X_{3} \\
\left(R^{2}=0.9704\right)
\end{gathered}
$$

The concentration of albedo $\left(X_{3}\right)$ had a significant negative linear effect $(P<0.05)$ on the flavor followed by a negative quadratic effect on the acid concentration $\left(X_{1} X_{1}\right)(P<0.05)$. The regression model adjusted to the experimental results (Table 6 ) shows significant value.

The unfamiliarity of the peculiar flavor and aroma of exotic fruits may be a factor that influences the sensory acceptance of flavored industrialized fruit (Perfeito et al., 2017), which explains the tasters' preference for gabiroba preserves with less pulp/sugar ratio.

Regarding texture, Figure 3, smaller amounts of albedo and citric acid were observed and higher pulp/ sugar ratio obtained higher scores. According to equation 4 , the concentration of citric acid $\left(X_{1}\right)$ and albedo $\left(X_{3}\right)$ had a negative effect on the texture. Table 6 shows a significant value for lack-of-fit $(P<0.05)$ in the regression model adjusted to experimental results.

$$
\begin{gathered}
\text { Texture }=22.3-30.2 X_{1}-7.7 X_{2}-1.98 X_{3}+ \\
+7.4 X_{1} X_{1}+11.6 X_{1} X_{2}-0.44 X_{1} X_{3}+1.29 X_{2} X_{3} \\
\left(R^{2}=0.6218\right)
\end{gathered}
$$

The formulations with higher averages did not have albedo added, however, they may have achieved an adequate and pleasant texture for the tasters due to the proportion of pulp and sugar present in the formulation.

Pectin present in the gabiroba fruit itself (Barbieri et al., 2019) may also have contributed to the texture of preserves. The pulp used for the production 
(A)

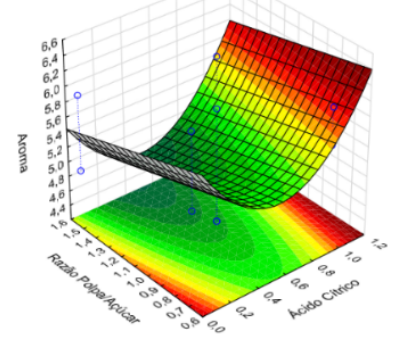

(B)

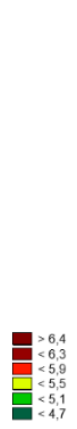

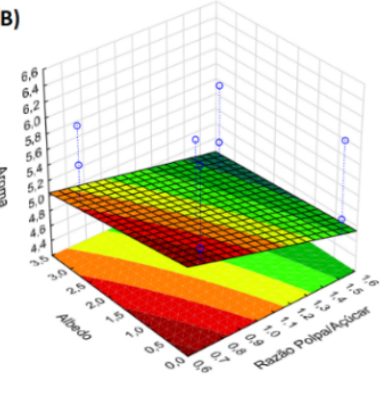

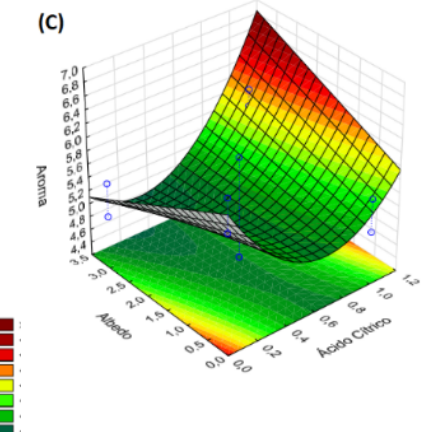

Fig. 1. Response surface of the aroma attribute as a function of the pulp/sugar ratio $\times$ acid citric $(\mathrm{A})$, albedo $\times$ pulp/sugar ratio (B), albedo $\times$ citric acid (C)

(A)

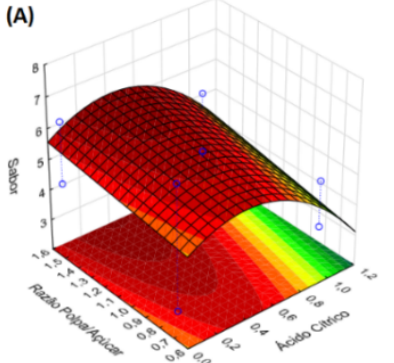

(B)

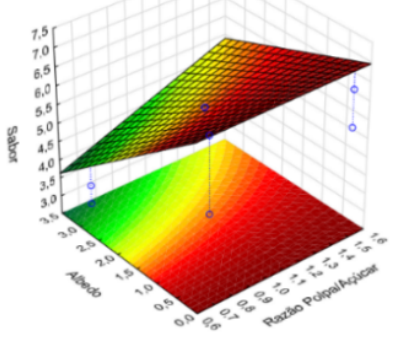

(C)

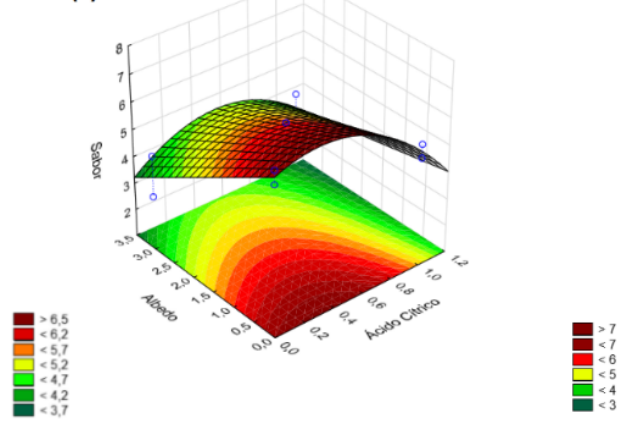

Fig. 2. Response surface of the flavor attribute as a function of the pulp/sugar $\times$ citric acid ratio (A), albedo $\times$ pulp/sugar ratio $(\mathrm{B})$, albedo $\times$ citric acid $(\mathrm{C})$

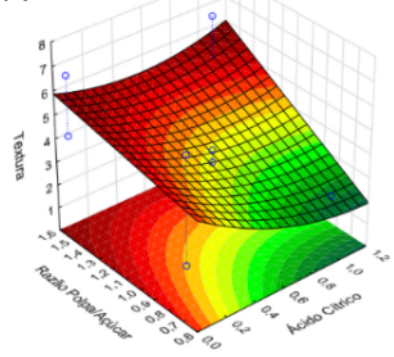

(B)

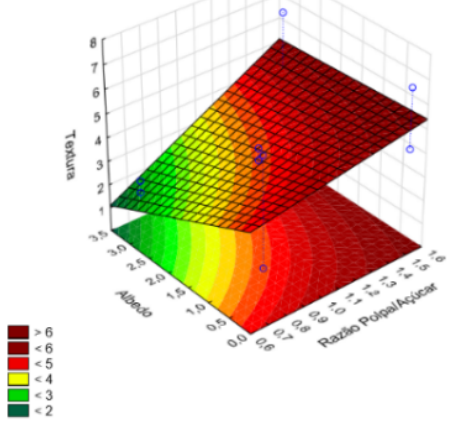

(C)

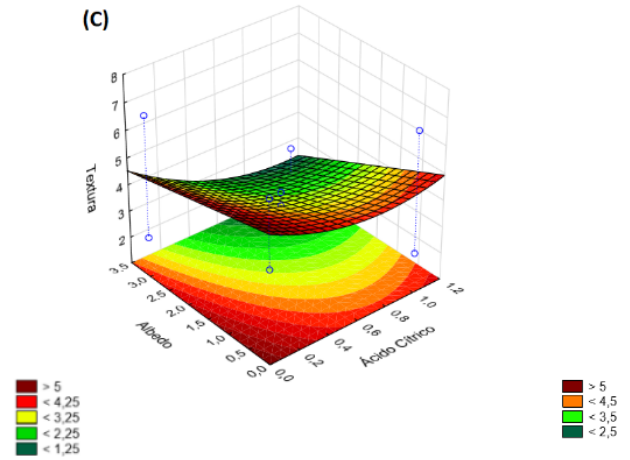

Fig. 3. Response surface of the texture attribute as a function of the pulp/sugar $\times$ citric acid ratio (A), albedo $\times$ pulp/sugar ratio (B), albedo $\times$ citric acid (C)

of preserve is the whole fruit, and fruit peels contain higher levels of acidity, dietary fiber and bioactive compounds than the pulp. The addition of high levels of fiber can increase the water holding capacity, forming a very viscous and firm textured gel (Teixeira and Novello, 2020). 
(A)

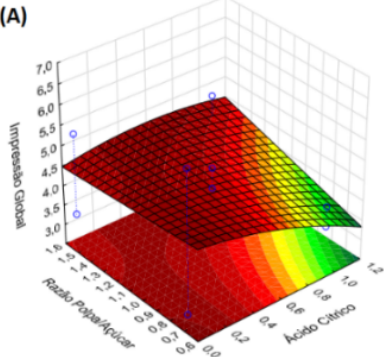

(B)

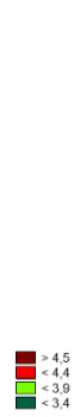

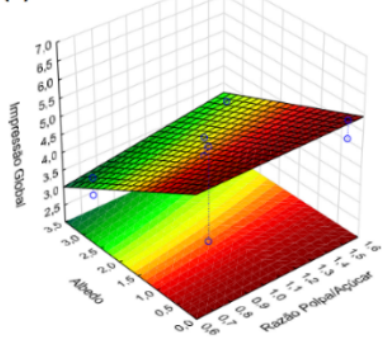

(C)

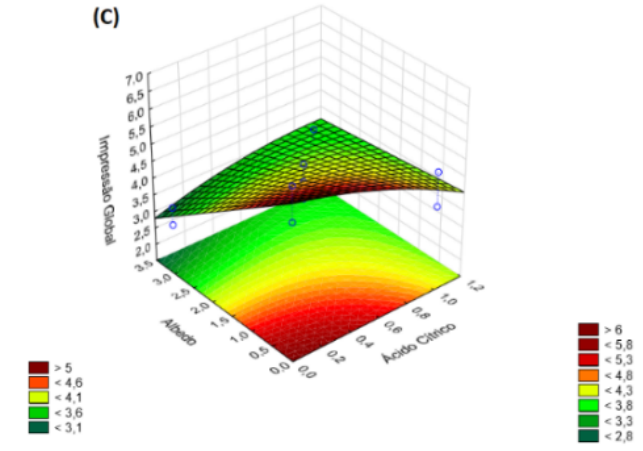

Fig. 4. Response surface of the global impression attribute as a function of citric acid $\times$ pulp/sugar ratio (A), albedo $\times$ pulp/ sugar ratio $(\mathrm{B})$, albedo $\times$ citric acid $(\mathrm{C})$

Regarding the global impression (Fig. 4), it was observed that the optimized formulations are those that presented lower levels of acid, albedo and pulp/ sugar ratio. The regression equation for the global impression of preserves is in equation 5:

Global impression $=15.31-9.6 X_{1}-4.4 X_{2}-1.551 X_{3}-$

$$
\begin{gathered}
-2.41 X_{1} X_{1}+5.81 X_{1} X_{2}+0.889 X_{1} X_{3}+0.323 X_{2} X_{3} \\
\left(R^{2}=0.9039\right)
\end{gathered}
$$

The albedo concentration had a greater influence in relation to the global impression, and the higher the albedo concentration, the lower the score obtained in this regard. The concentration of acid $\left(X_{1}\right)$, albedo $\left(X_{3}\right)$ and the pulp/sugar ratio $\left(X_{2}\right)$ had a negative linear effect on the global impression of the product followed by a negative quadratic effect on the acid concentration $\left(X_{1} X_{1}\right)$. The regression model adjusted to the experimental results of the global impression (Table 6) shows non-significant values for the sources.

According to the analysis of variance of the parameters of the sensory analysis, the albedo only had a significant influence on the flavor. It can be inferred, therefore, that the flavor is a highly relevant attribute for the global impression of the product.

The regression equation for the frequency of consumption is shown in equation 6 .

$$
\begin{gathered}
\text { Consumption frequency }=9.8-7.7 X_{1}-2.06 X_{2}- \\
-1.03 X_{3}+1.87 X_{1} X_{1}+1.45 X_{1} X_{2}+0.778 X_{1} X_{3}+ \\
+0.161 X_{2} X_{3} \\
\left(R^{2}=0.5937\right)
\end{gathered}
$$

In Figure 5, it can be seen that the optimum region regarding the frequency of consumption was obtained in formulations with lower amounts of albedo, citric acid and pulp/sugar ratio. The characteristics found in formulation 8 corroborate the result found in relation to the global impression of the product and also the effects presented in equation 6 , where the concentration of citric acid $\left(X_{1}\right)$ and albedo $\left(X_{3}\right)$ and pulp/sugar ratio $\left(X_{2}\right)$ had a negative linear effect.

The result of the low frequency of consumption of gabiroba preserves can be explained due to the fact that $55.55 \%$ of the tasters had never consumed the fruit previously, which reflects the consumers' opinion about the processed product since it maintains the characteristics close to those of the raw material.

Regarding the purchase intention (Fig. 6), it is possible to observe that formulations with lower levels of albedo are desirable, corroborating the consumer's preference for texture (Fig. 3) and flavor (Fig. 2).

The regression equation for the purchase intention of the preserves is:

$$
\begin{gathered}
\text { Purchase intention }=4.39-6.5 X_{1}+1.21 X_{2}- \\
-0.283 X_{3}+2.67 X_{1} X_{1}+0.667 X_{1} X_{3}-0.323 X_{2} X_{3} \\
\left(R^{2}=0.7745\right)
\end{gathered}
$$

The concentration of citric acid $\left(X_{1}\right)$ and albedo $\left(X_{3}\right)$ had a negative linear effect on the flavor purchase intention followed by a positive linear effect on the pulp/sugar ratio $\left(X_{2}\right)$. The regression model adjusted to the experimental results (Table 6) shows significant 
(A)

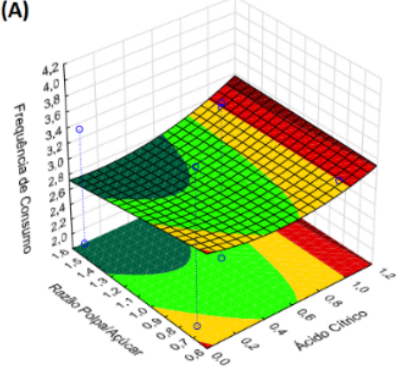

(B)

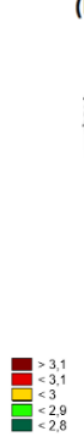

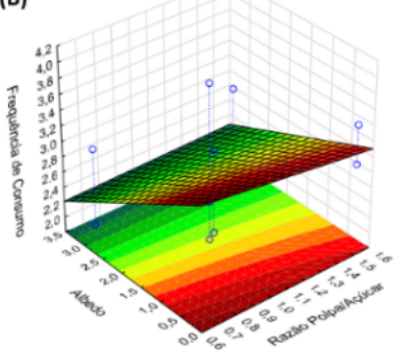

(C)

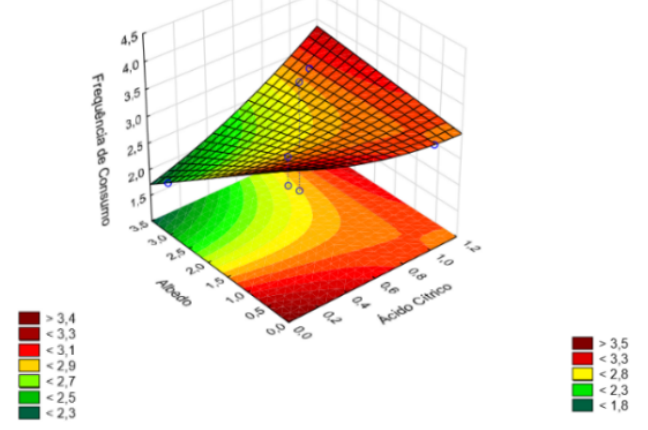

Fig. 5. Response surface of the frequency of consumption (attitude) as a function of the pulp/sugar $\times$ citric acid ratio (A), albedo $\times$ pulp/sugar ratio $(B)$, albedo $\times$ citric acid $(C)$

(A)

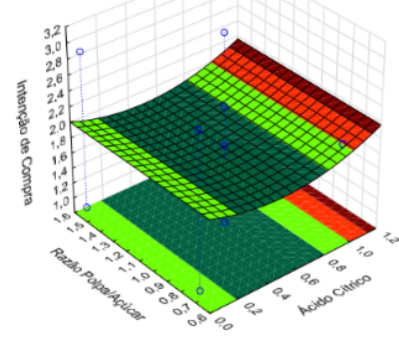

(B)

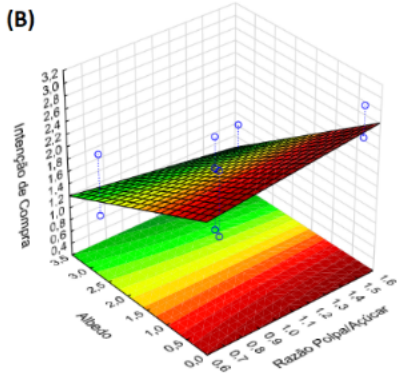

(C)

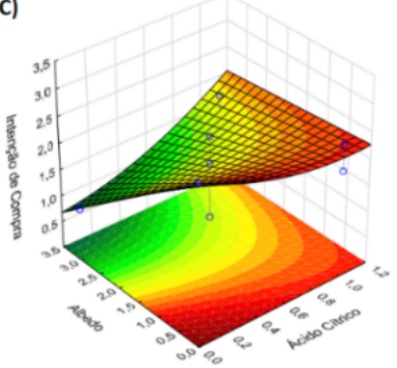

䈉

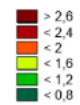

Fig. 6. Response surface of purchase intention as a function of pulp/sugar $\times$ citric acid ratio (A), albedo $\times$ pulp/sugar ratio (B), albedo $\times$ citric acid (C)

value. ANOVA for the purchase intention regression model is provided in Table 6.

From all the attributes, formulation 8 stood out, therefore, it was the formulation most appreciated by the tasters.

\section{CONCLUSION}

The gabiroba was adequate for its use in the production of preserves, which would facilitate its consumption regardless of the seasonality of the fruit, as well as the use of its nutrients all year round. The consumers' preference was observed for formulations with a lower pulp/sugar ratio, indicating the impact of the consumer's unfamiliarity with the fruit on his decision to consume it even if processed.

\section{REFERENCES}

Aguiar, A. O. de, Rodrigues, D. dos S., Souza, A. R. M. de, Soares, C. M. da S., Ibiapina, A., Filho, A. A. de M., ..., Martins, G. A. de S. (2019). Use of passion fruit's albedo as a source of pectin to produce Araticum use of passion fruit's albedo as a source of pectin to produce araticum (Annona crassiflora Mart.) preserves. Chem. Eng. Trans., 75 (January). https://doi.org/10.3303/CET 1975038

Barbieri, S. F., Amaral, S. C., Ruthes, A. C., Petkowicz, L. O., Kerkhoven, N. C., Silva, E. R. A., Silveira, J. L. M. (2019). Pectins from the pulp of gabiroba (Campomanesia xanthocarpa Berg): Structural characterization and rheological behavior. Carbohydr. Polym., 214(15), 250-258. 
Silva, D. L., Fonseca Leal, G., Silva Sousa, H. M., Tenório Pereira, C. M., Moreira Silva, J. F., Peluzio, J. M., de Souza Martins, G. A. (2021). Optimization of the preserved processing of all parts of the gabiroba fruit (Campomanesia adamantium (Cambess.) O. Berg) using response surface methodology. Acta Sci. Pol. Technol. Aliment., 20(4), 399-416. http://dx.doi.org/10.17306/J.AFS.2021.0891

Bekele, M., Satheesh, N., Sadik, J. A., Sc, M. (2020). Screening of Ethiopian mango cultivars for suitability for preparing jam and determination of pectin, sugar, and acid effects on physico-chemical and sensory properties of mango jam. Sci. African, 7, e00277. https://doi. org/10.1016/j.sciaf.2020.e00277

Box, G. E. P., Draper, N. R. (1987). Empirical model-building and response surfaces. New York: John Wiley.

Brasil (1998). Portaria n 27, de 13 de janeiro de 1998. Brasília: Agência Nacional de Vigilância Sanitária.

Brasil (2001). Resolução da Diretoria Colegiada-RDC n ${ }^{\circ} 12$ de 02 de janeiro de 2001. Brasília.

Brasil (2003). Resolução anvisa/ms rdc n 360, de 23 de dezembro de 2003. Brasília: Agência Nacional de Vigilância Sanitária.

Brasil (2012). Resolução n 54, de 12 de novembro de 2012. Brasília: Agência Nacional de Vigilância Sanitária.

Brasil (2019). Resolução da diretoria colegiada-rdc $n^{\circ} 331$, de 23 de dezembro de 2019. Brasília: Agência Nacional de Vigilância Sanitária.

Correia, L. F. M., Faraoni, A. S., Pinheiro-Sant'Ana, H. M. (2008). Efeitos do processamento industrial de alimentos sobre a estabilidade de vitaminas. Alim. Nutr., 19(1), $83-95$.

Curi, P. N., Tavares, B. S., Almeida, A. B., Pio, R. (2017). Characterization and influence of subtropical persimmon cultivars on juice and jelly characteristics. Anais Acad. Brasil. Ciênc., 89(2), 1205-1220. https://dx.doi. org/10.1590/0001-3765201720160101

Dias, J. D. M., Abreu, V. K. G., Pereira, A. L. F., Lemos, T. de O., Santos, L. H. dos, Silva, V. K. L. da, Mota, A. S. de B. (2019). Desenvolvimento e avaliação das características físico-químicas e da aceitação sensorial de doce em massa de cupuaçu. Bolet. Centr. Pesq. Proc. Alim., 36(1), 1-10.

Goldoni, J., Giacobbo, C. L., Galon, L., Zarzzeka, C., Uberti, A., Lugaresi, A. (2019). Physicochemical characterization of fruits of Campomanesia guazumifolia (Cambess.) O. Berg (Myrtaceae). Acta Sci., Biol. Sci., 41(e45923), 1-8. https://doi.org/10.4025/actascibiolsci. v41i1.45923

IAL (2008). Normas analíticas do Instituto Adolfo Lutz: métodos químicos e físicos para análise de alimentos (4th ed.). Brasília: Instituto Adolfo Lutz.

Lemos Jr, H., Brunelli, M. J., Lemos, A. L. A. (2011). Licopeno. Diagn. Tratament., 16(2), 71-74.
Nagata, M., Yamashita, I. (1992). Simple method for simultaneous determination of chlorophyll and carotenoids in tomato fruit. Nippon. Shokuhin Kogyo Gakkaisk, 39(10), 925-928.

Negri, T. C., Berni, P. R., Brazaca, S. G. C. (2016). Valor nutricional de frutas nativas e exóticas do Brasil. Biosaude, 12(2), 82-96.

Oliveira, T. T. B. de, Morais, R. A., Martins, G. A. de S. M., Marson, P. G., Teixeira, S. M. F. (2020). Processing of fruits of the cerrado in the form of integrals jellies. Rev. Agrar., 13(47), 130-140. https://doi.org/10.30612/agrarian.v13i47.8958

Paul, R. E., Duarte, O. (2012). Tropical fruits (3rd ed.). Barbados: University of the West Indies.

Perfeito, D. G. A., Corrêa, I. M., Peixoto, N. (2017). Elaboração de bebida com extrato hidrossolúvel de soja saborizada com frutos do Cerrado. Rev. Agric. Neotrop., 4(1), 21-27.

Schulz, M., Chim, J. F. (2019). Nutritional and bioactive value of Rubus berries. Food Biosci., 31, 33, 100438.

Silva, N. da, Junqueira, V. C. A., Silveira, N. F. de A., Taniwaki, M. H., Gomes, R. A. R., Okazaki, M. M. (2017). Manual de métodos de análise microbiológica de alimentos e água (5th ed.). São Paulo: Blucher.

Silva, I. G., Martins, G. A. de S., Borges, S. V., Marques, G. R., Regis, I. S. (2012). Influence of passion fruit albedo, citric acid, and the pulp/sugar ratio on the quality of banana preserves. Ciên. Tecnol. Alim., 32(2), 267-273. https://doi.org/10.1590/S0101-20612012005000038

Silva, M. L. S., Menezes, C. C., Portela, J. V. F., Alencar, P. E. B. da S., Carneiro, T. B. (2013). Carotenoids in frozen acerola pulps. Rev. Verde Agroecol. Desenvolv. Sustent., 8(1), 170-173.

Stone, H., Sidel, J. L. (2004). Sensory evaluation practices (3rd ed.). New York: Academic Press.

Teixeira, F., Novello, D. (2020). Physico-chemical, nutritional and sensory aspects of the addition of Citrus fruit byproducts in gelation products: a systematic review. Res. Soc. Develop., 9(3), e180932669. https://doi. org/10.33448/rsd-v9i3.2669

Tireki, S. (2017). Investigation of sensory and instrumental methods to predict shelf-life of jelly gums. Middle East Technical University.

Vieira, E. L. (2019). Fisiologia pós-colheita de frutos e hortaliças. Cruz das Almas: UFRB. 